\title{
Identificação de agentes infecciosos pulmonares em autópsias de pacientes com a síndrome da imunodeficiência adquirida
}

\author{
Identification of infectious agents in the lungs in autopsies of patients \\ with acquired immunodeficiency syndrome
}

\begin{abstract}
Sanivia Aparecida de Lima Pereira ', Denise Bertulucci Rocha Rodrigues', Dalmo Correia², Marlene Antônia dos Reis $^{3}$ e Vicente de Paula Antunes Teixeira ${ }^{3}$
\end{abstract}

\begin{abstract}
Resumo As afecções pulmonares são freqüentes em indivíduos infectados pelo vírus da imunodeficiência humana. Neste trabalho, procurou-se identificar através de histoquímica e imunohistoquímica, agentes infecciosos nos pulmões de indivíduos portadores da síndrome da imunodeficiência adquirida (SIDA), autopsiados entre março de 1990 e julho de 2000 na FMTM. Fragmentos de pulmão de 40 indivíduos com SIDA autopsiados foram analisados histologicamente. Foram identificados agentes infecciosos em 34 (85\%) casos dos 40 analisados, sendo que bactérias foram encontradas em $22(55 \%)$ casos. Entre os agentes fúngicos o Pneumocystis carinii foi encontrado em oito (19,1\%) casos; Cryptococcus sp em quatro (9,5\%), Histoplasma sp em dois $(4,8 \%)$ e Candida sp em um (2,4\%) caso. Detectou-se também associação entre Pneumocystis carinii, Citomegalovirus e Cryptococcus sp; CMV eToxoplasma gondii. Em cinco casos, Candida $\mathrm{sp}, \mathrm{CMV}$ e Pneumocystis carinii estiveram associados a bactérias. Entre as infeccões não bacterianas, os fungos foram os agentes infecciosos mais isolados dos pulmões em autópsias de indivíduos com SIDA, sendo o Pneumocystis carinii o mais freqüente.
\end{abstract}

Palavras-chaves: SIDA. Pulmão. Autópsia. Agentes infecciosos.

Abstract Lung diseases are frequently observed in individuals infected with HIV. The aim of this study was to identify infectious agents in the lungs in the autopsied individuals with AIDS performed between march 1990 and july 2000 at the school of medicine (Uberaba- Brazil), using histochemical and immunohistochemical techniques. Analysis was made on lungs obtained from 40 individuals with AIDS. Infectious agents were observed in 34 (85\%) cases of the 40 analyzed. Regarding fungis, Pneumocystis carinii was found in 8 (19.1\%)cases; Cryptococcus sp in 4 (9.5\%)cases, Histoplasma sp in $2(4.8 \%)$ cases and Candida sp in 1 (2.4\%) case. Association of Pneumocystis carinii, Citomegalovirus and Cryptococcus sp, was observed in one case, and in another, the association of CMV, and Toxoplasma gondii. There were 5 cases with Candida sp, $\mathrm{CMV}$ and Pneumocystis carinii associated with bacteria. In conclusion, this study shows that following bacterial agents fungi were the second most common infectious agents in post mortem examination of the lungs from patients with AIDS, being Pneumocystis carinii the most prevalent.

Key-words: AIDS. Lung. Autopsy. Infectious agents.

As afecções pulmonares são bastante prevalentes na evolução dos indivíduos infectados com o vírus da imunodeficiência humana, em todo o mundo ${ }^{3}$. Infecções respiratórias são responsáveis por cerca de dois milhões de mortes associadas à infecção pelo $\mathrm{HIV}^{3}$ e infecções bacterianas são as mais freqüentes nos pulmões de indivíduos com $\mathrm{SIDA}^{20}$. Dos agentes virais, o Citomegalovirus (CMV) é o mais freqüente em indivíduos com essa síndrome. A soroprevalência para esse vírus, na população em geral, varia em torno de $70 \%$, e nos pacientes infectados pelo HIV, em mais de $90 \%$. A imunodepressão, independente de sua origem, seja por terapêutica pós-transplante ou induzida pelo HIV, pode levar à reativação do CMV, presente em estado latente no hospedeiro ${ }^{27}$.

\footnotetext{
1 Disciplina de Patologia Geral da Universidade de Uberaba, Uberaba, MG. 2 Disciplina de Doenças Infecciosas e Parasitárias da Faculdade de Medicina do Triângulo Mineiro, Uberaba, MG, 3. Disciplina de Patologia Geral da Faculdade de Medicina do Triângulo Mineiro, Uberaba, MG.

Endereço para correspondência: Prof. Vicente de Paula Antunes Teixeira. Disciplina de Patologia Geral /FMTM. R. Frei Paulino 30, $38025-180$ Uberaba, MG.

Fax: 552134 312-6640

E-mail: vicpat@mednet.com.br

Recebido para publicação em 20/2/2002.
} 
A infecção fúngica também é causa comum de morbidade e mortalidade em pacientes imunodeprimidos. Essas infecções ocorrem primariamente em pacientes com neutropenia, induzida por quimioterápicos, na SIDA ou imunossupressão após transplante ${ }^{6}$. Centenas de espécies fúngicas têm sidos associadas com comprometimento pulmonar em humanos, como Cryptococcus neoformans, Candida sp, Pneumocystis carinii. No entanto, poucos agentes são realmente encontrados nos casos de pneumonite ${ }^{29}$.

O Cryptococcus neoformans e a Candida $s p$ correspondem aos agentes oportunistas de origem fúngica mais freqüentes na imunodepressão ${ }^{6}$. 0 Pneumocystis carinii não produz doença em indivíduos imunocompetentes, mas causa pneumonia grave em indivíduos com uma variedade de condições clínicas de debilitação ${ }^{16}{ }^{21}$. Até a ocorrência da epidemia da SIDA, a doença humana provocada pelo Pneumocystis carinii era limitada a lactentes subnutridos e pacientes imunossuprimidos por corticoterapia, terapia antineoplásica e transplantados. Entretanto, após o advento da SIDA, a pneumonia por Pneumocystis carinii é freqüentemente a primeira infecção oportunista diagnosticada nos indivíduos infectados pelo HIV-1 e constitui uma das principais causas de morte nesta doença2 479112328 .

Já foi encontrada, em material de a autópsia, associação entre Pneumocystis carinii e Histoplama capsulatum em pulmões de pacientes com SIDA. Estudos em autópsias de 25 pacientes com SIDA encontraram Histoplasma capsulatum em $68 \%$ dos casos, sendo que $48 \%$ foram identificados nos pulmões ${ }^{24}$.

A infecção por protozoários como o Toxoplasma gondii é menos freqüentemente encontrada nos pulmões de pacientes com SIDA. Entretanto, em alguns trabalhos já foi descrita infecção pulmonar por esse agente, por meio de autópsia, onde o pulmão aparece como o segundo órgão mais freqüentemente atingido 1214 .

O objetivo desse trabalho foi identificar agentes infecciosos nos pulmões de indivíduos autopsiados portadores da síndrome da imunodeficiência adquirida, por meio de histoquímica e imunohistoquímica.

\section{PACIENTES E MÉTODOS}

Foram analisados 296 protocolos de autópsias completas realizadas no Serviço de Patologia Geral da Faculdade de Medicina do Triângulo Mineiro, UberabaMG, no período de março de 1990 a julho de 2000, sendo selecionados 40 casos que correspondiam aos casos de indivíduos portadores de SIDA.

Os fragmentos de pulmão foram processados histologicamente, sendo realizadas as colorações de hematoxilina e eosina (HE) e prata metenamina-grocott.
Quando havia suspeita de bacilos álcool ácido resistentes (BAAR) realizava-se a coloração de Fitefaraco, sendo a coloração de Mucicarmim utilizada quando ao Grocott, suspeitava-se de Cryptococcus sp. A imunohistoquímica foi utilizada nos casos onde, após análise pela histoquímica rotineira, existiam dúvidas no diagnóstico. Os cortes foram analisados e realizada a pesquisa de agentes infecciosos através de um microscópio de luz comum.

\section{RESULTADOS}

Dentre os 40 casos analisados, encontrou-se agentes infecciosos em 34 (85\%) e em seis (15\%) nenhum agente foi identificado. Desses 34 casos, 21 (50\%) apresentavam bactérias não classificada e em um $(2,4 \%)$ caso encontrou-se bacilo álcool ácido resistente. Dos agentes fúngicos, o Pneumocystis carinii foi encontrado em oito $(19,1 \%)$ casos (Figura 1$)$, o Cryptococcus sp em quatro (9,5\%) (Figuras 2 e 3$)$, o Histoplasma $s p$ em dois $(4,8 \%)$ e Candida $s p$ em um
$(2,4 \%)$. Entre os vírus, o CMV foi encontrado em quatro $(9,5 \%)$ casos (Figuras 4 e 5$)$ e entre os protozoários o Toxoplasma gondii em um $(2,4 \%)$ caso (Figura 6$)$. Infecções concomitantes foram também observadas: Pneumocystis carinii, CMV e Cryptococcus sp (um caso), CMV e Toxoplasma gondii (um caso), Candida sp e bactéria não classificada (um caso), CMV e bactéria não classificada (dois casos), Pneumocystis carinii e bactéria não classificada (dois casos) (Tabela 1).

\section{DISCUSSÃO}

Dos 40 indivíduos portadores de SIDA em 34 casos, foram identificados agentes infecciosos no pulmão, sendo que as bactérias apareceram numa freqüência de $52 \%$, menor que os $77,3 \%$ encontrados em outro estudo realizado em Mumbai na India ${ }^{20}$.

Os agentes fúngicos apareceram numa freqüência de $35,8 \%$. Esses achados estão de acordo com outros estudos, em que as infecções fúngicas são causas comuns de morbidade em pacientes portadores de SIDA ${ }^{6}$.

Dos agentes fúngicos, o Pneumocystis carinii foi o mais freqüente, correspondendo a $8(19,1 \%)$ casos, sendo menor que os $26,8 \%$ descritos no pulmão de pacientes com SIDA em Porto Rico ${ }^{10}$. Este achado está diretamente relacionado com a progressão da SIDA, pois já foi demonstrado que a pneumonia por Pneumocystis carinii ativa a replicação do HIV no pulmão, uma vez que o pulmão representa um grande reservatório para esse vírus ${ }^{13}{ }^{17}$. Portanto, o Pneumocystis carinii pode ser responsável pela persistência e replicação do HIV, mesmo em períodos de não expressão clínica. Além disso, a grande replicação do HIV, durante infecções pulmonares, poderia influenciar no prognóstico da doença devido ao aumento da carga viral. Em um caso foi encontrada 

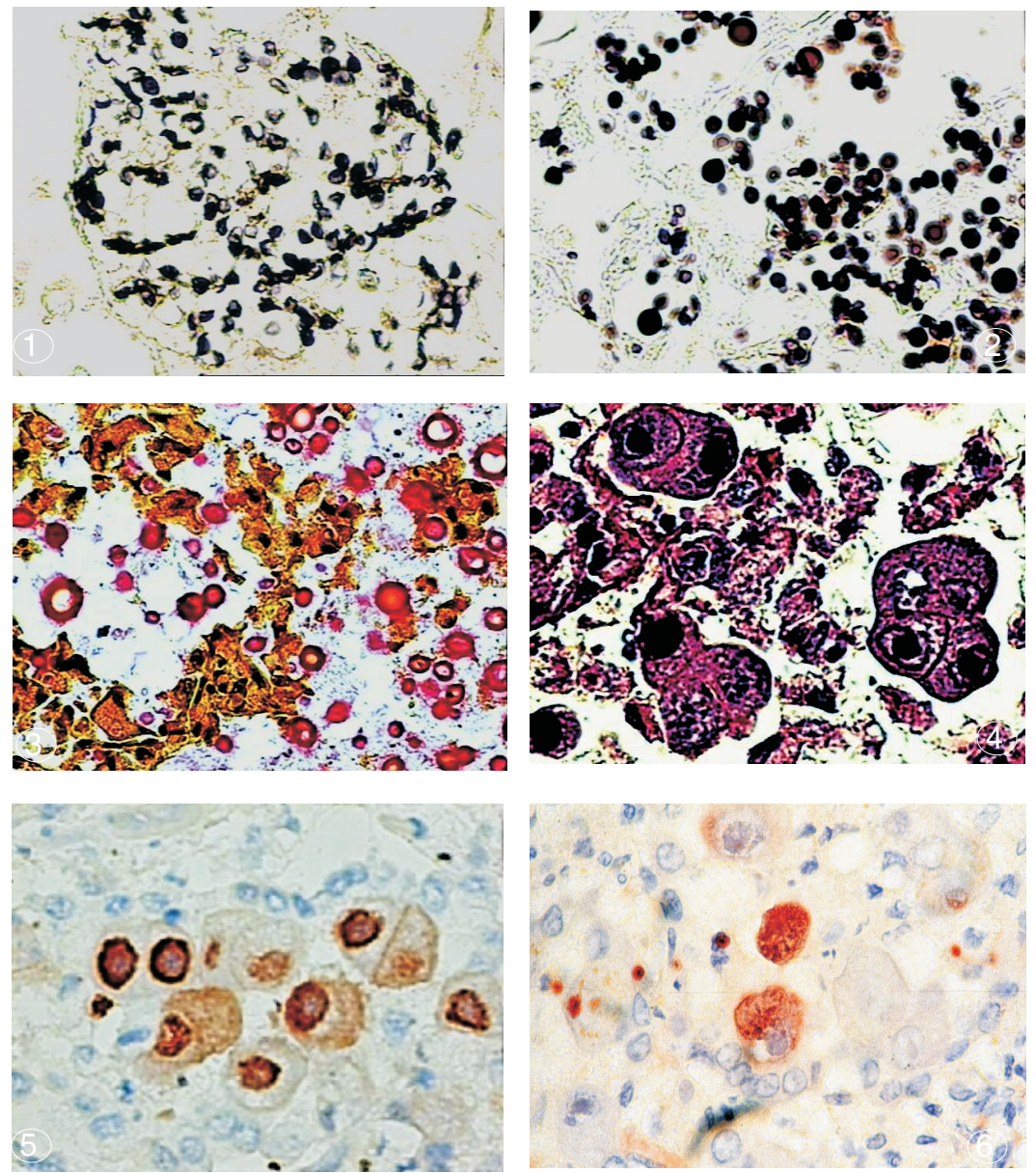

Cortes histológicos de pulmão obtidos de autópsias de indivíduos com SIDA

Figura 1 - Pneumocystis carinii (Prata Metanamina-Grocott X1280) Figura 2 Cryptococcus sp (Prata Metanamina-Grocott X1280). Figura 3 - Cryptococcus sp 5 - Citomegalovirus (Imunohistoquímica X1280). Figura 6 - Toxoplasma gondii e Citomegalovirus (Imunohistoquímica X100).

Tabela 1 - Distribuição dos agentes infecciosos no pulmão de 34 indivíduos com a síndrome da imunodeficiência adquirida autopsiados no Hospital Escola da Faculdade de Medicina do Triângulo Mineiro.

\begin{tabular}{llrr} 
& \multicolumn{3}{c}{ Agentes infecciosos } \\
\cline { 2 - 4 } Bactérias & identificados & $\mathrm{n}^{\circ}$ & $\%$ \\
& não classificada & 21 & 50,0 \\
Fungos & BAAR & 1 & 2,4 \\
& Pneumocystis carinii $^{*}$ & 8 & 19,1 \\
& Cryptococcus sp & 4 & 9,5 \\
Vírus & Histoplasma sp & 2 & 4,8 \\
Protozoários & Candida sp & 1 & 2,4 \\
\hline Total & citomegalovírus & 4 & 9,5 \\
\hline & Toxoplasma gondii & 1 & 2,4 \\
\hline
\end{tabular}

* Bacilo Álcool Ácido Resistente

O somatório e 42 agentes infecciosos, ultrapassa o total de 34 indivíduos com agentes infecciosos, porque em alguns casos encontrou-se mais de um agente. Pneumocystis carinii, CMV e Cryptococcus sp; CMV e Toxoplasma gondii, Candida e bactéria não classificada, um caso cada. CMV e bactéria não classificada; $P$. carinii e bactéria não classificada, dois casos cada. 
associação entre Pneumocystis carinii, CMV e Cryptococcus sp. Na pneumocistose é comum observar infecção concomitante por bactérias oportunistas, fungos ou vírus, especialmente o $\mathrm{CMV}$, que podem obscurecer a lesão causada pelo Pneumocystis carinii28. A associação entre Cryptococcus sp e Pneumocystis carinii foi demonstrada também em outros estudos ${ }^{5}$.

Encontrou-se também, associação entre CMV e Toxoplasma gondii. O Toxoplasma gondii foi descrito, em autópsias, em pulmões de indivíduos com SIDA, e a associação desse agente com o CMV também já foi demonstrada por autópsia, em pulmões de crianças com SIDA $^{1121430}$

Neste estudo, a freqüência de Cryptococcus $s p$ $(9,5 \%)$ foi menor que os $60 \%$ descritos por Balloul et al (1997), que fizeram cultura de lavados broncoalveolares de pacientes infectados pelo HIV 5 . Esta diferença talvez, deva-se ao método diagnóstico empregado, pois em um outro estudo, onde foram utilizados métodos semelhantes aos utilizados em nosso trabalho, esse agente foi encontrado em $6 \%$ dos pulmões de pacientes com SIDA autopsiados ${ }^{20}$.

A associação da cultura celular à histoquímica convencional aumenta a positividade diagnóstica das infecções oportunistas pulmonares em pacientes com SIDA. Utilizando histoquímica e cultura celular, alguns autores encontraram Histoplasma sp em $48 \%$ dos casos analisados em um estudo realizado nos pulmões de indivíduos autopsiados portadores de SIDA ${ }^{24}$.

A incidência de candidíase tem aumentado em pacientes imunodeprimidos, como na leucemia, linfoma maligno e na anemia aplásica. Nos indivíduos com
SIDA, a Candida spé mais freqüentemente encontrada no esôfago, seguida pelos pulmões e rins ${ }^{8}$. A baixa incidência de Candida sp $(2,4 \%)$ encontrada no presente trabalho pode ser devido ao uso de medicação com ação antifúngica (derivados triazólicos ou anfotericina B), como profilaxia secundária nos pacientes com SIDA.

O CMV foi em encontrado em $4(9,5 \%)$ casos, semelhante aos $8 \%$ encontrados em biópsia pulmonar de pacientes com SIDA $^{25}$ e aos $7 \%$ descritos no pulmão de pacientes com SIDA por meio de autópsia ${ }^{20}$. Estes autores concluíram que o CMV poderia ser considerado como possível causador da pneumonia em pacientes com SIDA avançada, especialmente se a infecção pelo CMV for documentada em outros órgãos.

Alguns estudos demonstraram que outros órgãos de pacientes com SIDA podem ser acometidos pelos agentes infecciosos aqui encontrados no pulmão, como o rim, onde foi descrita infecção por Cryptococcus sp e Histoplasma $s p^{18}$ e supra-renal, onde foram encontrados Cryptococcus sp, Histoplasma e CMV ${ }^{18}{ }^{19} 26$.

Após o uso em larga escala de terapia antiretroviral altamente eficaz (HAART), a incidência de infecções oportunistas nos pacientes com SIDA sofreu drástica redução, o que tem proporcionado aumento considerável da sobrevida e redução da morbidade ${ }^{1522}$.

Portanto, verificou-se que além dos agentes bacterianos, os fungos são mais comumente encontrados nos pulmões de indivíduos com SIDA, sendo que desses o Pneumocystis carinii foi o mais freqüente. A infecção por esse fungo indicaria um mau prognóstico, pois como já dito, o Pneumocystis carinii pode induzir a replicação do HIV.

\section{AGRADECIMENTOS}

Agradecemos à Fundação de Ensino e Pesquisa de Uberaba (FUNEPU), à Fundação de Amparo à Pesquisa do Estado de Minas Gerais (FAPEMIG), ao Conselho Nacional de Desenvolvimento Cientifico e Tecnológico (CNPq) e à Universidade de Uberaba (UNIUBE).

\section{REFERÊNCIAS BIBLIOGRÁFICAS}

1. Al-Kassab AK, Habte-Gabr E, Mueller WF, Azher Q. Fulminant disseminated toxoplasmosis in an HIV patient. Scandinavian Journal of Infectious Diseases 27:183-185, 1995

2. Alvarez F, Bandi V, Stager C, Guntupalli KK. Detection of Pneumocystis carinii in tracheal aspirates of intubated patients using calcofluor-white (Fungi-Fluor) and immunofluorescence antibody (Genetic Systems) stains. Critical Care Medicine 25:948952, 1997

3. Ashley EA, Johnson MA, Lipman MC. Human Immunodeficiency Virus and respiratory infection. Current Opinion in Pulmonary Medicine 6:240-245, 2000.

4. Aslanzadh J, Stelmach PS. Detection of Pneumocystis carini with direct fluorescence antibody and calcofluor white stain. Infection 24:248-250, 1996.

5. Balloul E, Couderc LJ, Molina JM, Cahite I, Volff M, Saimot AG, Caubarrere I. Pulmonary cryptococcosis during HIV infection. 15 cases. Revue des Maladies Respiratoires 14:365-370, 1997.
6. Cannoly JJ, Mcadams HP, Erasmus JJ, Rosado-de-Christenso, ML. Opportunistic fungal pneumonia. Journal of Thoracic Imaging 14:51-62, 1999

7. Eisen D, Ross BC, Fairbairn J, Warren RJ, Baird RW, Dwyer B. Comparison of Pneumocystis carinii detection by toluidine blue $\mathrm{O}$ staining, direct immunofluorescence and DNA amplification in sputum specimens from HIV positive patients. Pathology 26:198-200, 1994.

8. Furata I. Candida. Rinsho Byori. Japanese Journal of Clinical Hematology 48:1044-1050, 2000.

9. Goldenberg DM, Sharkey RM, Udem S, Vagg R, Levine GM, Conte P, Swayne LC, Hansen HJ, Cunniff D, Anton J. Immunoscintilography of Pneumocystis carinii pneumonia in AIDS patients [see comments]. Journal of Nuclear Medicine 35:10281034, 1994.

10. Gomes MA, Fernandez DM, Otero JF, Miranda S, Hunter R. The shape of the HIV/AIDS epidemic in Puerto Rico. Revista Panamericana de la Salud Publica 7:377-383, 2000. 
11. Gracia JD, Miravitlles M, Mayordomo C, Ferrer A, Alvarez A, Bravo C, Vendrell M. Empiric treatments impair the diagnostic yield of BAL in HIV-positive patients. Chest 111:1180-1186, 1997.

12. Hofman OP, Bernard E, Michiels JF, Thyss A, Le Fichoux Y, loubiere R. Extracerebral toxoplasmosis in the Acquired Immunodeficiency Syndrome (AIDS). Pathology, Research and Practice 189:894-901, 1993.

13. Israel-Biet D, Cadranel J. HIV replication and pulmonary opportunistic infections. Revue des Maladies Respiratoires 15:247-254, 1998.

14. Jautzke G, Sell M, Thalmann U, Janitschke K, Gottschalk J, Schurmann D, Ruf B. Extracerebral toxoplasmosis in AIDS. Histological and immunohistological findings based on 80 autopsy cases. Pathology, Research and Practice 189:428-436, 1993.

15. Kaplan JE, Hanson DL, Jones JL, Dworkin MS. Viral load as an independent risk factor for opportunistic infections in HIV infected adults and adolescents. AIDS 15: 1831-1836, 2001.

16. Kitada K, Oka S, Kimura S, Shimada K, Nakamura Y. Diagnosis of Pneumocystis carinii pneumonia by $5 \mathrm{~S}$ ribosomal DNA amplification. Journal of Protozoology 38:90S-91S, 1991.

17. Koziel H, Kim S, Reardon C, Li X, Garland R, Pinkston P, Kornfeld $\mathrm{H}$. Enhanced in vivo human immunodeficiency virus-1 replication in the lung of human immunodeficiency virus-infected persons with Pneumocystis carinii pneumonia. American Journal of Respiratory and Critical Care Medicine 160:2048-2055, 1999.

18. Laguna-Torres VA, Reis MA, Menegaz RA, Pelá GA, Prata A. Alterações anatomopatológicas renais em indivíduos com a síndrome da imunodeficiência humana. Revista da Sociedade Brasileira de Medicina Tropical 31:465-472,1998.

19. Laguna-Torres VA, Reis MA, Correia Filho D, Silva-Vergara ML, Prata A. Alterações renais clínico-laboratoriais em pacientes com a Síndrome da Imunodeficiência Adquirida em relação aos achados anatomopatológicos. Revista da Sociedade Brasileira de Medicina Tropical 33:207-215, 2000.
20. Lanjewar DN, Duggal R. Pulmonary pathology in patients with AIDS: an autopsy study from Mumbai. HIV Med 2:266-271, 2001.

21. Murray PR, Rosenthal KS, Dobayashi GS, Pfaller MA. Microbiologia Médica. Guanabara Koogan, Rio de Janeiro, 1998.

22. Poundstone KE, Chaisson RE, Moore RD. Differences in HIV disease progression by injection drug use and by sex in the era of highly active antiretroviral therapy. AIDS 15:1115-1123, 2001.

23. Rigole P, Basset D, Dedet JP. Diagnostic biologique de la pneumocystose. Evaluation et interet d'une nouvelle technique d'immunofluorescence directe. Pathologie Biologie 45:19-23, 1997.

24. Rocha MM, Severo LC. Histoplasmose disseminada em pacientes com síndrome de imunodeficiência adquirida (SIDA). Estudo de 25 casos. Revista do Instituto de Medicina Tropical de São Paulo 36: 167-170, 1994.

25. Rodrigues-Barrada MC, Stool E, Musher DM, Gathe J Jr, Goldstein J, Genta RM, Yoffe B. Diagnosing and treating cytomegalovirus pneumonia in patients with AIDS. Clinical Infectious Disease 23:76-81, 1996.

26. Rodrigues DBR, Reis M, Teixeira V, Silva-Vergara M, Correia Filho D, Adad S, Lazo J. Pathologic findings in the adrenal glands of autopsied patients with Acquired Immunodeficiency Sydrome. Pathology, Research and Practice 198: 25-30, 2002.

27. Salmon-Ceron D. Primary and secondary prevention of cytomegalovirus infections in immunocompromised patients. Annales de Medicine Interne 148:246-54, 1997.

28. Samuelson J, von Lichtenberg F. Doenças infecciosas. In: Cotran RS, Kumar V, Robbins SL (eds) Robbins Patologia Estrutural e Funcional 5 a edição, Guanabara Koogan, Rio de Janeiro, p. 269335, 1996.

29. Saubolle MA. Fungal pneumonias. Seminars in Respiratory Infections 15:162-177, 2000.

30. Yparraguirre IT, Sant'ana CC, Lopes VG, Madi K. Necroscopic study of 14 children with AIDS and pneumonia. Revista da Associação Médica Brasileira 47:129-136, 2001. 